\title{
Tendenze attuali delle teorie della letteratura
}

\author{
Costanzo Di Girolamo
}

Qualsiasi bilancio della situazione attuale della teoria letteraria finisce per essere, nella sostanza, un bilancio della storia delle teorie letterarie di tutto il ventesimo secolo, nel senso che sarebbe praticamente impossibile guardare a episodi isolati, a correnti particolari, a singole personalità, senza inserirli in una tradizione le cui premesse risalgono a molto tempo fa. La teoria letteraria, del resto, nasce con il ventesimo secolo: essa rappresenta una maniera specifica, e che non ha precedenti nel passato, di guardare alla letteratura. Vediamo anzitutto, brevemente, perché.

Le origini delle teorie letterarie contemporanee affondano le loro radici nella seconda metà del diciannovesimo secolo, con i movimenti dell'arte per l'arte (e si tratta di movimenti di artisti e di scrittori, dal simbolismo francese al futurismo italiano e russo, non di teorici). La definitiva acquisizione di una visione laica dell'esistenza, il relativismo morale, la specializzazione delle scienze, deprivano l'arte letteraria di funzioni che per secoli aveva avuto: oltre che veicolo di evasione fantastica o di trasporti emotivi, e anzi spesso proprio attraverso essi e grazie ad essi, per secoli l'arte letteraria è stata uno strumento di conoscenza del mondo e di organizzazione del sapere, ha proposto modelli di comportamento, quadri di realtà da comprendere e commentare, fedi e ideologie.

Non più, tutto questo, a partire da una certa data. Nel 1857, il difensore di Gustave Flaubert, Maître Marie-Antoine-Jules Sénard, aveva tentato di tutto per dimostrare alla giuria del Tribunale correzionale di Parigi che il romanzo del suo cliente, pubblicato in rivista l'anno prima, era un libro di irreprensibile moralità. Più perspicaci di lui si mostrarono i giudici nella loro sentenza: in base al loro senso morale, trovarono Madame Bovary un romanzo discutibile e in alcune parti «di un realismo volgare e scioccante», ma mandarono assolti l'editore, il tipografo e l'autore sulla base del riconoscimento delle qualità letterarie dell'opera. Il processo a Flaubert è una data significativa non tanto nella storia dell'affrancamento dell'arte dalla censura, come di solito si dice, quanto piuttosto nella storia della graduale conquista, da parte dell'arte, di una sua autonomia dal resto del mondo: di una emancipazione che per certi aspetti rappresenta una vittoria, per altri avrebbe avuto i suoi costi e le sue contropartite.

Questo nuovo modo di guardare all'arte e alla letteratura troverà presto le sue teorizzazioni e segnerà, in effetti, la nascita della teoria della letteratura. A 
volere essere più drastici, si può dire che segnerà la nascita stessa della letteratura. Dalla nostra prospettiva di fine del secondo millennio, quello di letteratura ci appare come un concetto ovvio, come qualcosa che va da sé, come una nozione universale, al riparo da qualsiasi relativismo storicistico: le opere di Omero, di Dante, di Llull, di Cervantes, di Shakespeare sono ovviamente tutte opere letterarie, e insieme con un numero incalcolabile di altre opere, scritte in una lingua qualsiasi, costituiscono quello che si chiama, e che per noi è, la letteratura.

Ma questo termine, fino almeno alla fine del diciottesimo secolo, designava (per esempio in francese, in italiano, in castigliano) non le opere, ma il ceto degli intellettuali e inoltre la carriera, le istituzioni letterarie (in latino, com'è noto, litteratura è un calco di Quintiliano sul greco grammatikê). Questo significa che fino a una certa data molto avanzata della Modernità non esisteva un termine specifico sotto cui comprendere scritture in versi e in prosa, queste ultime a loro volta distinte in scritture d'invenzione e scritture documentarie, e poi opere di teatro, la storiografia, un certo tipo di saggistica, e così via: tutti tipi di testi che per noi oggi sono, indiscutibilmente, letteratura; come per noi rientra nella letteratura anche quella che si chiama (erroneamente e con una palese contraddizione terminologica) la letteratura orale (l'oralità non ha niente a che vedere con la littera, con la scrittura), per esempio i poemi omerici, il Cantar de Mio Cid, il Romancero vecchio e nuovo, cioè tutta una serie di testi composti oralmente o per iscritto, ma comunque destinati all'esecuzione orale, indipendente dal libro manoscritto o a stampa. Si può forse dire che fino a una certa data esistevano dei generi ben definiti, con i loro sottogeneri: la poesia lirica, la poesia narrativa, il romanzo in versi o in prosa, il poema cavalleresco (in Spagna i romanzi di cavalleria), ecc. Ma, com'è noto, a partire dalla rivoluzione del Romanticismo si assiste alla graduale dissoluzione e alla definitiva confusione dei generi: molte forme di poesia, già alla fine del diciannovesimo secolo, si distinguono dalla prosa solo perché i righi tipografici non arrivano al margine destro della pagina, in assenza di una struttura metrica, della rima, di un lessico specifico, ecc. Si può insomma affermare che è solo a partire da quando oggetti fino a poco prima chiaramente classificabili entro generi o tipi di produzione artistica precisi si confondono l'uno con l'altro e, peggio ancora, si confondono con oggetti che non hanno nulla a che vedere con la sfera artistica (si pensi al lavandino di Marcel Duchamp), solo allora nasce l'esigenza di afferrare la specificità dell'arte, la sua essenza, la sua natura, cioè di riconoscerla e di definirla. In assenza di tratti normativi forti, del riferimento costante alle regole della tradizione, quella di letteratura diventa così una nozione debole, virtualmente aperta, che richiede l'individuazione della sua natura, dei suoi caratteri specifici.

Di qui anzitutto un paio di caratteristiche delle teorie letterarie del ventesimo secolo, che le distinguono da quanto altro potrebbe essere rubricato come teoria letteraria per i secoli passati.

La prima, è il carattere non prescrittivo della riflessione contemporanea sulla letteratura, a differenza delle poetiche antiche, medievali, rinascimenta- 
li e premoderne, che avevano tutte, senza eccezioni, un carattere normativo. Prima del Novecento, chi parla di letteratura inevitabilmente parla di come si deve fare letteratura, non di come funzionano o di come sono costruiti i testi letterari, né, tantomeno, di che cosa sia intrinsecamente letterario e che cosa no. La teoria della letteratura contemporanea invece osserva e descrive i meccanismi della letteratura, non indica nessun modello. Anche questo atteggiamento è in stretto rapporto con i movimenti artistici e letterari di fine secolo e dell'inizio del Novecento: a partire da una certa data, è vietato vietare; le arti, soprattutto le avanguardie, non conoscono più regole o canoni di valore, e si assiste al trionfo dello sperimentalismo. La teoria rispecchia l'esistente, non pretende di correggerlo o di modificarlo.

La seconda caratteristica, e forse più importante, delle teorie letterarie contemporanee è la loro indipendenza dall'estetica, dalla filosofia del bello, del piacevole: la ricerca dello specifico letterario e la descrizione dell'oggetto letterario non entra quasi mai nel merito del valore estetico, della riuscita e delle qualità dell'opera. Anche quando l'estetica è tirata in ballo, come fa l'estetica della ricezione (ma il movimento della Scuola di Costanza va anche, e forse più appropriatamente, sotto il nome di teoria della ricezione), la validità estetica dell'opera è presupposta, non è oggetto di argomentazione. Nel suo noto libro del 1967 sulla storia letteraria come provocazione, Jauss ci ricorda che l'effimero successo di Fanny di Ernest Feydeau, apparso a pochi mesi di distanza da Madame Bovary, fu abbastanza rapidamente travolto da quello del romanzo di Flaubert, che venne a modificare l'orizzonte di attesa del pubblico; ma Jauss, oltre a sottolineare la nuova tecnica narrativa di Flaubert (l'impassibilità) rispetto a quella vecchio-stile di Feydeau, non ci spiega per quali qualità o pregi estetici Madame Bovary riuscì a imporre un nuovo orizzonte di attesa al suo pubblico.

La teoria della letteratura, dunque, non propone regole e non giudica: fin dalla sua nascita sembra fondarsi come una disciplina puramente descrittiva.

Le tappe storiche principali delle teorie letterarie del ventesimo secolo sono abbastanza note, anche perché gli studi più importanti sono stati tradotti in diverse lingue; in particolare, molto è stato tradotto in italiano e in castigliano. Tuttavia un semplice itinerario cronologico attraverso la bibliografia non darebbe conto di una situazione molto più intricata: molti autori sono stati tradotti e conosciuti tardi, altri hanno portato in giro per il mondo le loro idee (è il caso di Jakobson), altri ancora (o interi movimenti) sono stati travolti dal dirigismo culturale (i formalisti russi, Bachtin) e quindi hanno avuto una certa risonanza solo in tempi relativamente recenti.

Il ruolo di pionieri spetta indiscutibilmente ai formalisti russi, un movimento le cui attività (durate all'incirca dal 1915 alla fine degli anni venti) si svilupparono di pari passo con l'avanguardia futurista. Sono i formalisti russi a individuare e a definire l'oggetto della teoria letteraria in termini che restaranno sostanzialmente invariati in tutta quella che si potrebbe chiamare la tradizio- 
ne formalistica (strutturalismo ceco, strutturalismo francese, neoretorica, semiotica; e nella tradizione formalistica potrebbe essere anche compreso il New Criticism anglo-americano): "oggetto della scienza della letteratura», scriveva Jakobson nel 1921, «non è la letteratura, ma la letterarietà, cioè ciò che di una data opera fa un'opera letteraria». Questa limpida dichiarazione programmatica faceva piazza pulita delle varie modalità (per esempio la biografia, la psicologia, la sociologia, l'approccio estetico) in cui si era dispersa fino ad allora la critica accademica russa e non solo russa. Nella loro ricerca della letterarietà, i formalisti si schierano dalla parte delle discipline sperimentali, parlano di "scienza della letteratura", non di critica letteraria o di studio della letteratura. Abbiamo detto che i formalisti si formano e vivono gomito a gomito con i futuristi, cioè con una delle prime avanguardie del Novecento che faceva della sperimentazione e della provocazione la sua bandiera. In realtà l'altro polo costante di riferimento del formalismo fu (ed è rimasto per quasi mezzo secolo) la linguistica teorica, la cui nascita si può simbolicamente far coincidere con la pubblicazione postuma del Cours de linguistique générale di Ferdinand de Saussure, nel 1916. E dunque nel linguaggio che i formalisti individuavano lo specifico della poesia, della letterarietà. Si tratta, ai loro occhi, di un linguaggio particolare, di un linguaggio, come dirà nel 1960 Jakobson, dominato dalla cosiddetta "funzione poetica», che consisterebbe nell'enfasi sul messaggio verbale in quanto tale, non per quanto comunica. Questa formula venne poi ripresa più volte e perfino estremizzata, fino alla demenziale affermazione del Gruppo $\mu$ (si ricorderà forse la loro Rhétorique générale, del 1970), secondo il quale «la parola poetica si squalifica come atto comunicativo. In effetti, essa non comunica nulla, o piuttosto non comunica che se stessa», un modo di vedere la poesia che avrebbe mandato in bestia poeti di ogni epoca, da Lucrezio a Dante a Ausias March fino ai nostri contemporanei.

Abbiamo detto che la tradizione formalistica prescinde dall'estetica. Ciò non significa tuttavia che non esistano vistose affinità tra i postulati del formalismo e la più importante estetica filosofica del nostro secolo, cioè l'idealismo crociano. Per cominciare, la nozione stessa di autonomia dell'arte. Per Croce come per i formalisti la poesia è del tutto aliena da finalità pratiche, è pura forma in cui si risolvono i contenuti, si identifica con l'espressione linguistica; per Croce come per i formalisti, infine, la "poesia» (la «letterarietà» di Jakobson) non va cercata unicamente nelle opere che rispondono a precisi canoni letterari: la poesia e la letterarietà sono insomma delle qualità intrinseche di certi oggetti, e non conoscono né codici né regole; e sintomatico è anche l'annullamento di qualsiasi barriera tra verso e prosa. Senza comunicare tra loro, l'estetica idealistica e il formalismo slavo delineavano, all'incirca nello stesso arco di tempo, l'una su basi rigorosamente estetiche, l'altro su basi linguistiche, una concezione dell'arte sostanzialmente affine, che poi affonda le sue remote origini nel Romanticismo. E non va dimenticato che quella idealistica è stata la corrente critica dominante nella cultura italiana per più di mezzo secolo (solo in parte affiancata da quella marxista a partire dagli anni cinquanta) prima dell' avvento della critica strutturalistica. 
Non abbiamo purtroppo il tempo per seguire le tappe di questa che è sicuramente la più ampia corrente di teoria letteraria del Novecento: quella che abbiamo appunto chiamato la "tradizione formalistica» e che, come ho detto prima, dal formalismo russo arriva fino allo strutturalismo, alla neoretorica e alla semiotica degli anni Sessanta e Settanta, irrigidendo sempre più i propri dogmi e la propria visione della letteratura. Certamente, sarebbe sbagliato fare di ogni erba un fascio. Molti formalisti russi furono, oltre che agguerriti teorici, critici finissimi e robusti storici letterari; l'esponente più importante dello strutturalismo praghese (anni trenta), Jan Mukarovský, ortodossamente formalista in alcuni scritti, si pose ben presto il problema del valore estetico; e probabilmente anche personalità come quelle di Bachtin e di Lotman, apparentemente lontane dai padri formalisti, sarebbero impensabili senza il retroterra del formalismo storico. Abbiamo accennato all'insensatezza delle posizioni del Gruppo $\mu$, ma negli stessi anni in Francia Gérard Genette avviava una paziente descrizione della scrittura letteraria che non può non essere definita filologica; in Italia, le correnti strutturalistiche e semiologiche alternavano prese di posizione teoriche tutt'altro che emozionanti alla pratica della filologia, della ricostruzione storico-letteraria e di una saggistica di alto livello (com'è noto, in Italia alla teoria letteraria si sono dedicati principalmente i filologi). Nel frattempo, tendenze della critica virtualmente agli antipodi del formalismo cedevano in maniera disarmante ad esso. In Italia, per esempio, il fiorente filone (fino agli anni Sessanta) della critica marxista o sociologica era pronto a riporre le armi davanti al rigore della critica strutturalistica incarnata da scienziati della letteratura in camice bianco: si pensi alle professioni di fede di Franco Fortini o di Alberto Asor Rosa. Anche la critica freudiana (ancora in Italia si pensi all'importante caso di Francesco Orlando) finiva per servirsi della critica formale e per usarla almeno come strumento di analisi. I metodi strutturalistico-semiologici erano entrati intanto nelle scuole, e forse fino a tutti gli anni ottanta hanno costituito la vulgata critica, a consacrazione definitiva del loro trionfo su qualsiasi altra impostazione dello studio letterario, a cominciare da quelle impostazioni degli studi letterari che non ritengono necessario il ricorso a una teoria.

Poi viene la crisi... La crisi che compare perfino nel titolo dell'ultimo libro di Cesare Segre, Notizie dalla crisi, del 1993. Ma di che crisi si tratta, e, esiste veramente una crisi?

Una svolta importante avviene sicuramente alla fine degli anni sessanta. Dobbiamo quindi fare, cronologicamente, un passo indietro: i fenomeni e le date ovviamente si accavallano, perché le nuove idee hanno bisogno di un periodo più $o$ meno lungo di incubazione.

Non mi sembra imprudente mettere in rapporto alcuni cambiamenti nello studio della letteratura con le ventate rivoluzionarie, molto astrattamente rivoluzionarie, del '68. Del resto, sono svolte teoriche che avvengono nelle università europee e americane, non più nei caffe di Pietroburgo e di 
Mosca frequentati da poeti e da anarchici; sono svolte che avvengono nelle stesse università dove gli studenti proponevano criticamente nuovi modi di guardare alla realtà e ai loro stessi oggetti di studio. È indiscutibile che il '68 abbia inciso vistosamente, se non nella storia politica e sociale, almeno nella storia delle mentalità e dei comportamenti di questo secolo, e forse nella storia della cultura. È semmai curioso, o forse triste, che uno dei maggiori movimenti di teoria letteraria che può essere ricondotto a quegli anni e a quei fermenti, cioè il decostruzionismo, abbia avuto per maestro un uomo con alle spalle un taciuto passato nazista: il belga Paul de Man, come è stato provato dopo la sua morte, era durante la guerra un collaborazionista nel suo paese occupato dai tedeschi. A questo maestro di teoria, se non proprio di pratica e di vita, va almeno riconosciuto il merito di aver saputo cavalcare la tigre e di circondarsi per lungo tempo del fervore dei progressisti e dei marxisti. Diverso è certamente il caso della teoria della ricezione e del suo fondatore: le voci circolate alcuni anni fa sui trascorsi di Hans Robert Jauss ventenne nelle SS (quello delle SS era un corpo di volontari) sembrano destituite di ogni fondamento e ad esse non può essere dato alcun credito. Resta però il fatto che la fisionomia intellettuale di Jauss ha ben poco a che vedere con quella di un uomo della sinistra: in un certo senso, Jauss è riuscito a cavalcare la tigre assai meglio di un progressista come Harald Weinrich o di un marxista come Erich Köhler (violentemente contestato dai suoi studenti), entrambi a lui molto vicini.

La teoria della ricezione rispondeva in effetti all'esigenza di oltrepassare i confini asfittici entro cui era insegnata la letteratura nelle università tedesche, dominate dal metodo idealistico e da quello formalistico. Nello stesso anno 1967, oltre al libro di Jauss sulla storia della letteratura come provocazione, fu pubblicato anche un breve ma importante saggio, Per una storia letteraria del lettore, appunto di Weinrich, uno studioso appartato e originale (anche lui un filologo romanzo come Jauss), a conferma del fatto che certe idee erano nell'aria: secondo entrambi, l'opera letteraria ha un ineludibile termine di confronto nel pubblico, un pubblico storicamente e culturalmente individuato, che determina scale di valori e che in qualche misura incide sugli stessi sviluppi della produzione letteraria.

Nel libro sulla storia letteraria, Jauss teorizza che il valore e la carica innovativa di un'opera si misurano in rapporto all'orizzonte di attesa di un determinato periodo storico; nozione, questa di orizzonte di attesa, ripresa da Gadamer, ma già presente in Husserl e in Heidegger. In questo e in altri lavori degli stessi anni, Jauss rivela ancora punti di contatto con la tradizione formalistica nel privilegiare gli aspetti «innovativi», perfino «devianti», della grande arte. Sennonché, già in questi studi l'innovazione viene vista non solo come scarto di ordine formale, ma anche come capacità di mettere in discussione le convenzioni sociali dominanti. L'orizzonte di attesa non va quindi inteso unicamente come una 
sorta di codice letterario in possesso dei destinatari, ma rientrano in esso gli usi e le norme sociali, il modo di vedere il mondo e di rapportarsi con gli altri che è specifico di un certo momento storico. In virtù della sua funzione «socialmente formativa», la letteratura contribuisce secondo Jauss all'emancipazione dell'umanità dai suoi vincoli naturali, religiosi e sociali.

Come si vede, la musica cambia... Jauss risolleva infatti una questione, quella della funzione formativa dell'arte, che era stata da tempo accantonata negli studi letterari. Anche un altro esponente di spicco della Scuola di Costanza, l'anglista Wolfgang Iser, pone alla base della sua attenta e rigorosa fenomenologia della lettura una serie di preoccupazioni di tipo sostanzialmente etico e sociale. Per Iser è fondamentale, nel processo della comunicazione letteraria, l'interazione tra testo e lettore. Ora (semplifico al massimo), nell'immagine che un'efficace rappresentazione letteraria dà del mondo il lettore riconosce elementi familiari ma depragmatizzati, vale a dire rimossi dal loro contesto originale: il lettore ha cioè «la possibilità di percepire un sistema in cui era stato fino ad allora preso, e questa consapevolezza sarà tanto maggiore se la validità di queste norme è negata. [...] Se il lettore è indotto a formulare le cause che sono alla base della messa in questione del mondo, questo significa che deve andare oltre quel mondo, in modo da poterlo osservare dall'esterno. Ed è qui che risiede la vera funzione della letteratura».

$\grave{E}$ evidente, solo a volere considerare l'opera critica e teorica di Jauss e di Iser, come la teoria della ricezione apra lo studio della letteratura a problematiche di carattere, se non propriamente estetiche in senso stretto, indubbiamente filosofiche, ricollegandosi alla tradizioni di pensiero, particolarmente ricche in Germania, della fenomenologia e dell'ermeneutica. Alla Scuola di Costanza va riconosciuto anzitutto il merito di avere posto in primo piano il problema dell'interpretazione (problema per la verità, come vedremo subito, mai venuto meno nella tradizione americana), che era stato del tutto accantonato dagli indirizzi formalistici: come abbiamo osservato, $\mathrm{i}$ formalisti non si chiedono che cosa significhi o che cosa voglia dire un'opera ma ne descrivono la forma, le strutture manifeste o soggiacenti. I teorici della ricezione ripropongono le questioni del significato letterario e della funzione della letteratura, ma mettendo in rapporto questi problemi con il pubblico, e con un pubblico storicamente individuato.

Va ricordato che questa attenzione ai destinatari si può cogliere, negli stessi anni (negli anni settanta in particolare), anche negli Stati Uniti, con la cosiddetta "critica orientata verso il lettore» (reader-oriented criticism o reader-response criticism), benché questo indirizzo non abbia mai rappresentato un movimento compatto quanto piuttosto un'area di raduno durante e dopo la disintegrazione dello strutturalismo.

Ma l'idillio tra testo e lettore non doveva durare a lungo. Posto che un testo è del tutto inerte se non è interpretato, se non è fatto vivere o rivivere dall'inter- 
pretazione del lettore, si solleva a questo punto il problema di quale o di quali interpretazioni siano legittime o corrette, se esista un limite alle interpretazioni possibili e, addirittura, se non esista nessuna, nemmeno una sola interpretazione che si possa considerare impossibile. È su queste problematiche che, a partire dalla fine degli anni sessanta, si avvia in America un acceso dibattito.

Una distinzione suggerita all'interno della recente critica americana è tra ermeneutica negativa e ermeneutica positiva: da un lato i sostenitori dell'indecidibilità del testo e dell'illimitatezza delle interpretazioni (i decostruzionisti maggiori, minori e minimi; Holland e altre personalità); dall'altro, i sostenitori dell'autorità autoriale (il testo significa quello che l'autore intendeva fargli significare) e quindi dell'univocità dell'interpretazione (Hirsch, Booth, Abrams e altri). Le implicazioni delle due assunzioni, e i rischi connessi, sono abbastanza evidenti. Le posizioni monistiche ad oltranza (un testo ha un'unica interpretazione possibile) ricadono in quella che già Wimsatt e Beardsley avevano bollato, fin dal 1954, come «intentional fallacy», cioè la presunzione che l'interpretazione possa fondarsi sull'intenzione dell'autore, che peraltro è spesso tutt'altro che evidente e talvolta inconoscibile. D'altra parte, l'ermeneutica negativa, o pluralistica, è inevitabilmente orientata verso il nichilismo. Dall'ammissibilità di diverse interpretazioni si passa, con Paul de Man (il suo libro più importante, Blindness and Insight è del 1971) e con altri decostruzionisti (soprattutto Harold Bloom: The Anxiety of Influence, 1971; A Map of Misreading, 1975), a quella che si potrebbe chiamare l'ermeneutica del fraintendimento, del misreading, perfino (secondo Paul H. Fry) l'ermeneutica della distrazione (l'interpretazione efficace o illuminante avviene quando si allenta la concentrazione, l'attenzione).

La storia del decostruzionismo americano, in aperta crisi a partire dalla fine degli anni ottanta, è tuttora da fare seriamente e, per chi mai vorrà farla, questo non sarà un compito facile. In realtà, più che di un movimento o di una corrente, il decostruzionismo può essere visto come un prolungato dibattito sulla teoria e sui metodi, più che sulla letteratura e tanto meno su testi letterari precisi, con massicce interferenze di tipo filosofico. Bisogna infatti ricordare che, fino alla fine degli anni sessanta, negli Stati Uniti per filosofia si intendeva quasi esclusivamente la tradizione della filosofia analitica, qualcosa di ben diverso da ciò che noi in Europa intendevamo, e continuiamo a intendere, per filosofia (gli stessi dipartimenti di filosofia erano e continuano a essere nelle università americane tutt'uno con i dipartimenti di matematica). A partire dalla fine degli anni sessanta, grazie anche alla costante presenza negli Stati Uniti di personalità come Foucault e soprattutto Derrida, che diventano ben presto degli habitués delle rotte atlantiche, si assiste in America a vere e proprie orge a base di filosofia; orge che si consumavano, ovviamente, non nei dipartimenti di filosofia (credo che Derrida non abbia mai messo piede in un dipartimento di filosofia americano), ma nei dipartimenti di inglese, di lingue (soprattutto di francese), di letterature comparate. Io personalmente ho assistito, quando insegnavo a Baltimora, ad alcune belle lezioni di Derrida sui presocratici che mi ricordavano quelle del mio professore del liceo (con tutta 
la stima per Derrida, avevo un ottimo professore di filosofia): solo dopo un po' di tempo ho capito che la cosa rivoluzionaria, e che attirava tanti studenti e colleghi, era semplicemente che si impartissero delle lezioni di storia della filosofia.

La difficoltà di assestare storicamente il decostruzionismo americano risiede proprio nel fatto che il dibattito comprende tutte le voci possibili in quegli anni: freudiani (soprattutto lacaniani), marxisti, femministe, ermeneuti, fenomenologi, nichilisti puri..., tutti orientati verso una critica della critica o verso la critica di tutto; la stessa validità dei testi letterari, per molti decostruzionisti, consisterebbe nel decostruire, nel negare, nel rovesciare se stessi, ovvero i loro significati apparenti o voluti dall'autore. Per tutti questi aspetti, il decostruzionismo si presenta forse come il ribaltamento simmetrico del formalismo strutturalistico-semiologico: alla presenza assoluta, chiusa e irrifrangibile del testo postulata da quest'ultimo, il decostruzionismo contrappone la dissoluzione del significato nella miriade di interpretazioni possibili e impossibili e nella volontaria o involontaria autodistruzione del testo. Alla battuta della studentessa di Stanley Fish (Fish non è un decostruzionista, ma un intelligente e abilissimo interlocutore dei decostruzionisti) che dà il titolo a una sua bella raccolta di saggi del 1980, Is There a Text in This Class? (si tratta di un aneddoto scherzoso: una studentessa chiede al professore se c'è un libro di testo per quel corso, ma la frase può essere intesa anche come una domanda sui metodi dell'insegnante, «si crede all'esistenza dei testi in questo corso?»), a questa battuta il decostruzionismo, nei vent'anni della sua breve esistenza, sembra non aver saputo o voluto rispondere.

In contrapposizione al formalismo all'europea o all'americana (cioè alla tradizione, lenta a morire, del New Criticism), si forma infine, a partire dall'inizio degli anni ottanta, l'ultima, almeno a tutt'oggi, corrente organizzata di teoria letteraria statunitense, il New Historicism, anche se, come vedremo subito, si tratta di una «teoria debole», per così dire (naturalmente, all'espressione «teoria debole» non attribuisco alcuna valutazione negativa). Fatto sta che la discussione teorica in America non langue affatto, anche se questa discussione ha ora come fine, paradossalmente, l'abolizione della teoria. Segnalo su questo indirizzo, per chi fosse interessato, il n. 8 (1993) della rivista "L'Asino d'oro» e in particolare il saggio introduttivo di Barbara Gastaldello, da cui ricavo alcune informazioni.

La paternità del termine New Historicism sembra da attribuire all'anglista americano Stephen Greenblatt, il fondatore della rivista "Representations», che lo avrebbe utilizzato per primo in un suo scritto del 1982, insieme con l'espressione "poetics of culture», che però non è stata ripresa da altri (Greenblatt parla di un «nuovo storicismo o di una poetica della cultura»). Il neostoricismo, ultima moda teorica negli Stati Uniti, presenta in realtà parecchie affinità con la corrente di studi britannica che va sotto il nome di cultural studies, risalente 
agli anni cinquanta-sessanta, che ha avuto parecchi proseliti in diverse università italiane, in particolare tra gli anglisti dell'Istituto Universitario di Napoli.

Come il decostruzionismo, anche il neostoricismo mira al superamento della specificità dello studio letterario. Più che come un metodo di critica letteraria in senso stretto, il neostoricismo può essere infatti considerato un indirizzo che ha per oggetto la storia della cultura in generale, i processi di comunicazione non solo verbale, la trasmissione del sapere e i rapporti tra il potere e le espressioni artistiche, i mass media, le forme della cultura di massa, le subculture giovanili, gli studi post-coloniali, i gender studies (cioè gli sviluppi della critica femminista e la neonata critica gay), ecc. Anche i riferimenti bibliografici di questi studiosi cambiano. Se la presenza di Foucault rimane costante rispetto al decostruzionismo, compaiono spesso in questi saggi, singolarmente, i nomi di due italiani abbastanza dimenticati in patria: Giambattista Vico e Antonio Gramsci. Forse il libro più bello prodotto in quest'ambito di studi, che peraltro precede di qualche anno la nascita dell'etichetta «neostoricismo", è quello del palestinese, naturalizzato americano, Edward Said, Orientalism, del 1978, una splendida storia della rappresentazione e dei pregiudizi che nel corso dei secoli l'Occidente ha elaborato dell'Oriente.

Nel suo complesso, il neostoricismo si presenta come un enorme calderone entro il quale sarebbe inutile andare alla ricerca delle nozioni di «teoria» o di «metodo». Al contrario, è proprio dall'interno di questo indirizzo che sono stati sferrati gli attacchi più duri alla teoria. Mi riferisco a un breve ma importante saggio di Stephen Knapp e Walter Benn Michaels, Against Theory, apparso nel 1982 in "Critical Inquiry», che originò un acceso dibattito. Knapp e Michaels sono fautori di un "nuovo pragmatismo», che metta da parte qualsiasi rigidità teorica e di metodo, a favore di una pratica della critica e dell'interpretazione svincolata da modelli preconcetti. La posizione di Knapp e Michaels veniva subito condivisa dallo stesso Greenblatt, che scriveva nel 1985: «Per me lo studio della letteratura è studio di opere contingenti, particolari, volute, immerse nella storia; se la teoria comporta inevitabilmente il desiderio di sfuggire dalla contingenza verso una sfera superiore, in cui i segni siano purificati dal fango della storia, allora io sono contro la teoria».

Per avviarci alla conclusione, torniamo alla crisi di cui parla Cesare Segre. Come abbiamo avuto modo di vedere, gli ultimi vent'anni hanno proposto un dibattito e una riflessione sulla letteratura vivace e di notevole qualità. A parte il fastidio che possono suscitare tutte le mode ripetitive (soprattutto all'interno del decostruzionismo, non si contano i libri puramente insensati), in questo arco di tempo si è venuto articolando un dialogo tra posizioni anche lontane tra di loro (per esempio tra teorici della ricezione e decostruzionisti, tra marxisti e neostoricisti), che ha avuto almeno il merito di mettere costantemente in discussione tutto. Anche posizioni fino a poco tempo fa ghettizzate o snobbate, come la critica marxista inglese, hanno trovato di recente maggiore atten- 
zione e ascolto. La tendenza generale è dunque quella di «complicare» il discorso sulla letteratura con implicazioni filosofiche, antropologiche, sociali, di storia della cultura nel senso più vasto. Da questo punto di vista, non mi pare che si possa parlare affatto di crisi, almeno in situazioni come quella americana, britannica, tedesca e francese. In realtà, la crisi di cui parla Segre è esclusivamente la crisi delle teorie e dei metodi forti, in particolare del metodo strutturalistico-semiologico, fino a poco tempo fa incontrastato e dominante (ma, non va dimenticato, metodi forti sono stati in passato anche la critica idealistica e un certo tipo di critica marxista). Se per teorie letterarie si intendono solo i metodi forti, allora è vero che questi sono in crisi e, per quanto mi riguarda, è una fortuna che lo siano.

Per la verità, la situazione in Italia è quella che è soprattutto perché, da almeno vent'anni, qualsiasi invito alla discussione, anche alla polemica, è stato lasciato cadere nel vuoto. Una tendenza della critica italiana è stata quella di procedere inglobando sincretisticamente tutto ciò che incontrava, fino al punto, ad esempio, che i semiologi si sono fatti portavoce in prima persona della teoria della ricezione (nata su basi antiformalistiche), di un autore come Bachtin, della culturologia di Tartu, e via dicendo. Solo con il decostruzionismo questa operazione, pure avviata da qualcuno, non è riuscita: singolarmente, Derrida e il decostruzionismo americano sono stati recepiti in Italia soprattutto e quasi esclusivamente dalla cultura cattolica. Ma interventi critici contro il formalismo e contro i modelli forti, risalenti alla metà degli anni settanta, fatti per esempio da Franco Brioschi, da Alfonso Berardinelli, da Cesare Cases, da Nicola Merola e, mi sia permesso di ricordarlo, da chi vi parla, questi interventi e questi inviti alla discussione semplicemente non hanno avuto risposta. Molti di noi pensano che riflettere sulla letteratura, fare teoria letteraria, non comporti l'elaborazione di procedure operative rigide a cui sottoporre i testi; non comporti cioè l'elaborazione di un "metodo»: il critico deve necessariamente sporcarsi, come dice Greenblatt, con il «fango» che i testi portano con sé, senza fermarsi al livello epidermicamente linguistico e formale (come facevano i formalisti) e probabilmente senza ignorare (come hanno invece fatto i decostruzionisti) che i testi letterari hanno un significato che va compreso, discusso, perfino contestato.

Tutto sommato, c'è da essere ottimisti. Forse è meglio la crisi, è meglio il dubbio di tante mal fondate certezze. 